\title{
ENTREVISTA CON LA DRA. MARTA CECILIA GUTIÉRREZ GIRALDO: LA FORMACIÓN DEL PROFESORADO BASADO EN PRÁCTICAS REFLEXIVAS
}

Fecha de recepción: 17/02/2017

Fecha de aceptación: 30/03/2017

Resumen: En esta entrevista se desarrolla el enfoque de formación docente basado en prácticas reflexivas. La Dra. Marta Cecilia Gutiérrez Giraldo menciona que la formación del profesorado debe realizarse desde campos de formación interdisciplinarios que sean comunes y partiendo de las características del profesorado; puesto que en la formación de docentes reflexivos, las prácticas educativas constituyen la base sobre la cual los profesores dialogan y analizan problemas reales que acontecen en sus aulas y proponen soluciones a estos. También, se menciona que el aprendizaje debe ser flexible considerando los ritmos de aprendizaje de los estudiantes. La Dra. Gutiérrez concluye afirmando que, las emociones son esenciales dentro del proceso de enseñanza y aprendizaje.

Palabras clave: Formación, enseñanza, aprendizaje, emociones.

\begin{abstract}
This interview develops the teacher training approach based on reflective practices. Dra. Marta Cecilia Gutiérrez Giraldo mentions that the training of teachers should be carried out from interdisciplinary training fields that are common and based on the characteristics of the teaching staff, being that in the training of reflective teachers, educational practices are the basis on which Teachers dialogue and analyze real problems events in their classrooms and propose solutions to them. He also mentions that learning must be flexible considering students learning rhythms. She concludes by stating that emotions are essential in the teaching and learning process.
\end{abstract}

Keywords: Training, teaching, learning, emotions.

\section{Presentación}

La Dra. Marta Cecilia Gutiérrez Giraldo es psicóloga y educadora especial de la Universidad de Manizales. Magister en Educación de la Universidad San Buenaventura de Bogotá y doctora en Ciencias Pedagógicas de la Universidad de la Habana. Actualmente es profesora titular de la Facultad de Educación de la Universidad Tecnológica de Pereira. Coautora del libro Prácticas educativas reflexivas y formación del profesorado, publicado por la Editorial Universidad Tecnológica de Pereira en el año 2015. 


\section{Alejandra Barquero Ruiz ( $A B)$ : ¿En que consiste el enfoque de formación docente basado en prácticas reflexivas?}

\section{Marta Cecilia Gutiérrez Giraldo (MCGG):}

El enfoque de la formación de profesores basado en prácticas reflexivas no es nuevo ni es novedoso. Lo novedoso es que nosotros en América Latina empezamos a generar otras posibilidades para hacer de la formación de los docentes una propuesta alterna a los enfoques tradicionales de capacitación. Lo que se hace tradicionalmente es que se les lleva un experto y ese experto le dice al profesor qué hay que hacer, entonces el profesor sale de la capacitación muy contento porque el experto le dijo que había que hacer, pero las cosas siguen igual cuando el profesor llega al aula a su práctica cotidiana, es decir no cambió nada. A principios del siglo XX, John Dewey, empezó a plantear esta propuesta de las prácticas educativas reflexivas y a lo largo del siglo $\mathrm{XX}$, se fueron dando experiencias tal

vez la más novedosa y que impactó más en el mundo fue la que se derivó de Shön en trabajos con médicos, arquitectos y músicos, en los cuales se dice que en el ejercicio de una profesión no es suficiente la teoría ni la acción por la acción, sino la relación teoríapráctica. Eso data de toda la discusión que hemos tenido a lo largo de la historia, si es la teoría o la práctica. Son estos enfoques que vienen desde las teorías constructivistas y otros desde las teorías críticas, las cuales nos dicen que uno no se forma solo leyendo y estudiando y, luego, realizando una práctica sin saber qué va a hacer y tampoco se forma con el hacer, porque tampoco es suficiente y desde esa reflexión en la teoría y la práctica es lo que se denomina formación en prácticas reflexivas que fue acuñada en la década de los 70 y 80 por Shön; por lo que, se genera todo un movimiento de transformación de la formación de profesionales llegando al ámbito educativo, generándose una serie de iniciativas o movimientos, uno de ellos lo lideramos nosotros, el cual se relaciona con la formación de los docentes en prácticas reflexivas y en ese movimiento lo que hacemos es que nosotros no volvemos a trabajar la formación de docentes desde capacitaciones; sino 
que, por un lado las hacemos más desde campos de formación que son interdisciplinarios y comunes y; por otro lado partimos de lo que son los profesores.

Los profesores cuando llegan a procesos de formación, nos llegan con concepciones, con prácticas, con experiencias. Ellos tienen cosas que contar, que aprender, que enseñar y que compartir, eso rompe con los enfoques tradiciones de formación y nos lleva por otro camino que es la formación en comunidades de indagación de práctica, de aprendizaje, otros hablan de comunidades de práctica, otros los diferencian de comunidades de aprendizaje, pero la base de eso está todo en una propuesta derivada de la teoría crítica que nos lleva a que nos involucremos, como ciudadanos, como profesores, como miembros de una comunidad comprometidos con el cambio, el cambio en nuestro quehacer profesional y a partir de eso nos encontramos con colegas que tienen las mismas dificultades, los mismos problemas o problemas similares y, en ese proceso podría haber o no invitado, pero ya el invitado no entra a decir qué hacer o cómo hacerlo, sino que el invitado entra como un facilitador, como un mediador y a través de ese proceso empezamos a conocer los distintos problemas que tenemos desde nuestras experiencias cotidianas como 3 profesores y desde ahí, entonces, hacemos búsqueda de problemas comunes, priorizamos problemas comunes y sobre esa priorización de problemas comunes nos conformamos voluntariamente en comunidades de aprendizaje, en comunidades de indagación y empezamos a buscar soluciones en las que todos aportamos, todos estudiamos, todos construimos, todos trabajamos, todos las llevamos a nuestros entornos y a partir de esas búsquedas comunes vamos compartiendo, vamos debatiendo y eso nos lleva a plantear propuestas de prácticas educativas donde repensamos todo nuestro quehacer desde los propósitos, para qué quiero hacer yo lo que estoy haciendo en el aula de clase, porqué lo quiero cambiar. Ya no es porque otro me dice qué estoy haciendo; sino porque yo, libre y voluntariamente en diálogo con otro, percibo la necesidad de cambio, voy y reviso con otros mis propias concepciones, mis propios imaginarios, mis propios problemas y los de otros y, vamos organizándonos para que busquemos soluciones a los problemas que tenemos en la práctica $\mathrm{y}$, desde ahí, empecemos a planear propuestas alternativas, propuestas didácticas alternativas que nos ayuden a transformarnos y transformar y, en eso

Revista Perspectivas: Estudios Sociales y Educación Cívica - No. 14

ISSN-L: 2215-4728 • enero-junio 2017 • pp. 1-6

DOI: http://dx.doi.org/10.15359/rp.14.1 
nos vamos volviendo bastante reflexivos porque aprendemos a hacer reflexión en la acción a través de la acción y sobre la acción.

La reflexión en la acción es aquella que nos dice que cuando estamos en un aula por más preparados que estemos, por más conocimientos que tengamos del campo, siempre habrá problemas porque nos encontramos con seres de carne y hueso. El estudiante posee conocimientos previos, los cuales hay que aprovechar y realizar adaptaciones, hay que ser flexible porque si no se es flexible en el aula, les estamos uniformando, haciendo del aprendizaje un aprendizaje rígido como si todos aprendiéramos de la misma manera, al mismo ritmo y esto hace que tengamos que ir siempre haciendo reflexión en la acción. Posterior a una acción educativa hay que reflexionar sobre la misma, esto implica que pensemos en lo que pasó, en lo que planificamos, en si se cumplieron los objetivos propuestos y desde ahí revisamos lo que se hizo en la acción.

\section{ABR: ¿Cuáles son los requisitos para el aprendizaje flexible según lo manifestado en su libro Prácticas educativas y la formación del profesorado?

MCGG: Esto implica cambiar los enfoques de enseñanza y aprendizaje, de evaluación, es así como la enseñanza tiene que cambiar y flexibilizarse y el aprendizaje también, eso implica que a la hora de planear un proceso de enseñanza y aprendizaje este tiene que respetar los ritmos de aprendizaje de los estudiantes. Ahí somos muy Vigotskyanos en aquello de trabajar el aprendizaje social y que unos estudiantes sirven de apoyo, sirven de compañeros para el aprendizaje de otros, hay estrategias por cantidades para dar esas ayudas pero todo se basa en el principio de ayudas ajustadas que implican que atendamos las necesidades de los estudiantes en el momento en que las tienen, que sean flexibles, variadas, oportunas y temporales. De lo que se trata es de educar para la autonomía, de ser capaces de flexibilizar la forma de enseñar y aprender, eso significa que los estudiantes van a ser capaces de ir cumpliendo con los objetivos propuestos y garantizar aprendizajes más significativos y duraderos. 
ABR: ¿Cómo desarrollar en el contexto latinoamericano una formación docente basada en prácticas reflexivas?

MCGG: Partiendo desde cada comunidad y cada contexto. No hay fórmulas tenemos que empezar por concientizarnos a nosotros mismos. Yo pienso que los gobiernos, las universidades y las instituciones gastan muchísimo dinero en procesos de formación que no terminan siendo formación, sino que se convierten en adiestramiento o capacitación, esto tiene más implicaciones y está más relacionado con la importancia que le damos a la educación, a la transformación de nuestros propios contextos y a pensar qué es la calidad de la educación en términos más prácticos. Es romper con esos procesos de formación desde arriba, es empezar desde abajo y hacer del profesor un intelectual, que piensa, que siente, que decide, que aporta a la comunidad, que transforma, porque siempre nos ven desde arriba y desde afuera, señalándonos como parte del problema, pero los docentes no somos el problema, somos parte de la solución.

\section{$A B$ ¿Y cuál sería el principal reto que según su experiencia podrían tener las universidades, en relación a esto que usted plantea?}

MCGG: Pues tienen que confiar en nosotros como profesores, porque echarle la culpa a los profesores es muy fácil y eso es lo que hemos hecho toda la vida, porque el gobierno culpa a las universidades, las universidades culpan a los profesores, los profesores señalamos a los estudiantes, los estudiantes culpan a la familia; entonces, como sociedad no hemos asumido responsabilidades en esta materia. El reto es que seamos capaces de repensar y asumirnos como intelectuales orgánicos, comprometidos con el cambio y eso nos lleva a pensarnos como intelectuales en transformación y transformadores.

Revista Perspectivas: Estudios Sociales y Educación Cívica - No. 14

ISSN-L: 2215-4728 • enero-junio 2017 • pp. 1-6

DOI: http://dx.doi.org/10.15359/rp.14.1 


\section{AB: ¿Cómo puede entenderse la relación entre cognición, emoción y aprendizaje dentro del enfoque de formación basada en prácticas educativas?}

MCGG: En la propuesta de formación de docentes basada en prácticas educativas reflexivas no se abandona nunca esa reflexión entre cognición, emoción y aprendizaje, porque es que lo que nosotros somos, hacemos y pensamos. Todo está permeado por las emociones, por los sentimientos. Los estudios de Damasio son claros al indicar que las emociones guían el juicio y la acción en ambientes educativos, además de destacar el papel de las emociones en la toma de decisiones y en el aprendizaje, al resaltar que es desde la cultura que se forma este. Si no vinculamos las emociones al aprendizaje, si dejamos que la escuela siga privilegiando el factor cognitivo, no vamos a poder asegurar un aprendizaje significativo. Cuando yo parto de la revisión de mis propias prácticas, yo tengo que revisar mis concepciones, mis imaginarios; tengo que ir a la práctica y hay emociones, cosas positivas y negativas. Una investigación doctoral que acabo de dirigir sobre la expresión 6 emocional en el aprendizaje lo muestra claramente, esta visualiza como las emociones del profesor en el aula terminan siendo muchas veces las emociones del estudiante y evidencia que cuando el profesor llega estresado a hablar de exámenes y otras cosas, la expresión emocional de los estudiantes cambia en el aula. Uno cree que no y, eso es muy impactante, pues ver como los estados emocionales de un profesor tienen mucho que ver con los estados emocionales del estudiante.

Revista Perspectivas: Estudios Sociales y Educación Cívica - No. 14

ISSN-L: 2215-4728 • enero-junio $2017 \bullet$ pp. 1-6

DOI: http://dx.doi.org/10.15359/rp.14.1 